\title{
EFECTO DEL REFINAMIENTO DE LA DESCRIPCIÓN DE LA RUGOSIDAD EN UNA APROXIMACIÓN 2D PARA UN RÍO DE MONTAÑA: UN CASO DE ESTUDIO
}

\section{ON THE EFFECT OF THE REFINEMENT OF THE ROUGHNESS DESCRIPTION IN A}

\author{
2D APPROACH FOR A MOUNTAIN RIVER: A CASE STUDY
}

\author{
Juan Sebastián Cedillo Galarza*1 ${ }^{*}$, Luis Manuel Timbe Castro ${ }^{1,2} 2^{\oplus}$, Esteban \\ Patricio Samaniego Alvarado ${ }^{1,2}$ [ y Andrés Omar Alvarado Martínez ${ }^{1,2}$
}

\author{
${ }^{1}$ Departamento de Recursos Hídricos y Ciencias Ambientales. Universidad de Cuenca, Av. 12 de Abril s/n, 010107, Cuenca, \\ Ecuador. \\ ${ }^{2}$ Facultad de Ingeniería. Universidad de Cuenca, Av. 12 de Abril s/n, 010107, Cuenca, Ecuador. \\ *Autor para correspondencia: sebastian.cedillog@ucuenca.edu.ec
}

\begin{abstract}
Resumen
La predicción de niveles de agua en ríos es importante para prevenir pérdidas económicas así como de vidas humanas causadas por inundaciones. Los modelos hidráulicos son comúnmente usados para predecir estos niveles de agua y tomar acciones para mitigar el daño debido a inundaciones. En la presente investigación, se analizó una aproximación 2D para resolver las ecuaciones promediadas en profundidad de Reynolds Average Navier Stokes (RANS), llamado Conveyance Estimation System (CES), para explorar sus capacidades predictivas. Este artículo presenta una ampliación del estudio realizado por Knight y col., 2009. De igual forma, en esta investigación se explora una caracterización más detallada del parámetro de rugosidad y del número de zonas de rugosidad produciendo diversos escenarios. Se evaluó el desempeño de cada escenario mediante diferentes funciones de ajuste usando curvas de descarga para comparación. La investigación muestra que el uso de una adecuada descripción de la rugosidad, como un factor de rugosidad calibrado para toda la sección transversal o un modelo de rugosidad para cantos rodados calibrado para el lecho junto con valores de rugosidad obtenidos en valores sugeridos por el CES para los bancos, produce resultados del modelo óptimos en un río de montaña.
\end{abstract}

Palabras clave: Sistema de estimación de capacidad de trasporte, Ríos de montaña, coeficientes de rugosidad. 


\begin{abstract}
The prediction of water levels in rivers is important to prevent economical as well as human losses caused by flooding. Hydraulic models are commonly used to predict those water levels and take actions to mitigate flooding damage. In this research, a 2D approach to solve the depth average Reynolds Average Navier Stokes (RANS) equations, called Conveyance Estimation System (CES), is analyzed to explore its capabilities for prediction. This article presents an extension of the study performed in Knight y col., 2009. More specifically, in this study, a more detailed characterization of the roughness parameter and the number of roughness zones is explored producing additional scenarios. The performance of each scenario is evaluated by means of different fitting functions using rating curves for comparison. The research shows that the use of an adequate roughness description, such as a roughness factor calibrated for the whole cross section or a boulder roughness model calibrated for the channel bed plus roughness values from the CES roughness advisor for banks, leads to optimal model results in a mountain river.
\end{abstract}

Keywords: Conveyance Estimation System, Mountain Rivers, roughness coefficient

Forma sugerida de citar: Cedillo-Galarza, J.S., Timbe-Castro, L.M., Samaniego-Alvarado, E.P. y AlvaradoMartínez, A.O. (2021). Efecto del refinamiento de la descripción de la rugosidad en una aproximación 2D para un río de montaña: un caso de estudio.. La Granja: Revista de Ciencias de la Vida. Vol. 33(1):92-103. http:/ / doi.org/10.17163/lgr.n33.2021.08.

IDs Orcid:

Juan Sebastián Cedillo Galarza: http:/ / orcid.org/0000-0001-6866-8937

Luis Manuel Timbe Castro: http:/ /orcid.org/0000-0001-5623-0487

Esteban Patricio Samaniego Alvarado: http:/ / orcid.org/0000-0002-8728-491X

Andrés Omar Alvarado Martínez: http:/ / orcid.org/0000-0002-9125-1221 


\section{Introducción}

Las inundaciones constituyen uno de los desastres naturales más peligrosos pues producen grandes pérdidas humanas y económicas (Douben, 2006; Camp, Whyte y Shaw, 2016). Desde el punto de vista de la modelización, la mitigación de los efectos de las inundaciones requiere el cálculo de los niveles de agua. Cuando se utilizan modelos hidrodinámicos 1-D, se deben usar las ecuaciones de Saint-Venant (Papanicolaou, Bdour y Wicklein, 2004), que contienen un parámetro de resistencia, el cual suele ser el parámetro de resistencia Manning (n). Sin embargo, este parámetro a veces no es lo suficientemente útil para la toma de decisiones. Por lo tanto, en esta investigación, nos enfocamos en una aproximación 2-D llamada CES (Conveyance Estimation System, por sus siglas en inglés) para estimar el nivel de agua en un área específica de un río de montaña.

Hay mucha información relacionada a esta aproximación 2D, como la geometría del río o los parámetros que aparecen en diferentes ecuaciones de modelado. Sin embargo, en esta investigación nos enfocamos en el factor $\mathrm{f}$ de rugosidad, el cual se puede estimar de diferentes maneras (Marcus y col., 1992). En la metodología se consideran las ecuaciones empíricas, que requieren variables que sean de fácil medición, fiables y que consideren los efectos de la resistencia en el área y cerca del área (Jarrett, 1985; Bathurst, 2002; Ferguson, 2007). Por lo general, las fórmulas disponibles para estimar el factor f pueden tener errores de aproximadamente 30\% porque se desarrollaron considerando la media de variaciones en múltiples áreas (Bathurst, 2002).

Además, las fórmulas obtenidas en "áreas específicas" para los ríos de montaña no son comunes, pues la fricción es el único o principal componente de resistencia (Bathurst, 2002; Romero, Revollo y Molina, 2010). Por lo tanto, su aplicación se limita a ciertos tipos de alcances (planos, sin vegetación y sin entrada de aire). Además, el factor de rugosidad implica diferentes elementos según la estructura del modelo: 1D, 2D o 3D. En los modelos 1D, el parámetro contiene una representación incorrecta de turbulencia (Bhola, Leandro y Disse, 2019), mientras que en algunos modelos $2 \mathrm{D}$ la representación de rugosidad no incluye turbulencia (Morvan y col., 2008). En esta investigación se analiza el desempeño de tres ecuaciones empíricas con datos recolectados en un río de montaña. Dos de ellas son expresiones semilogarítmicas mencionadas por Knight y col. (2009) y Romero, Revollo y Molina (2010) y el resto es una expresión exponencial obtenida en Bathurst (2002). Estas ecuaciones se explican detalladamente en el siguiente apartado.

Los ríos de montaña tienen algunas características que influyen en el factor $\mathrm{f}$ de rugosidad. La profundidad del agua (d) es comparable al material del lecho del río con una inmersión relativa d/D84 que oscila entre 4 y 10 (Bathurst, 2002). En consecuencia, el material del lecho contribuye más a la resistencia que lo que ocurre en los ríos planos (Jarrett, 1984). La distribución de velocidad en los ríos de montaña tiene forma de $\mathrm{S}$ en lugar del perfil semilogarítmico utilizado en los ríos de baja pendiente (Bathurst, 1985), debido a la presencia de rocas que tienen un diámetro mayor a $256 \mathrm{~mm}$, y menor a $4000 \mathrm{~mm}$ (Bunte y Abt, 2001). Existe una velocidad de flujo por debajo del nivel de las rocas y entre las rocas, así como altas velocidades por encima de las rocas. El patrón de velocidad es importante ya que existe una relación entre la velocidad y la resistencia (Wohl, 2000). Se observan efectos adicionales de las rocas debido al impacto del flujo de agua en su superficie saliente, así como la formación de corrientes detrás de ella (Jarrett, 1984). Además, Pagliara, Das y Carnacina (2008) demuestran que la interacción de la superficie del agua con las rocas incrementa a medida que aumenta la concentración de rocas.

El CES es un modelo bidimensional que resuelve las ecuaciones promediadas en profundidad de Reynolds Average Navier Stokes (RANS) en un área transversal. Estas ecuaciones sirven para estudiar la fricción utilizando un factor de rugosidad unitario (nl), el cual representa un segmento del área transversal. Además, el factor nl es un parámetro de resistencia obtenido con un amplio alcance donde los elementos de rugosidad son pequeños en relación a la profundidad del agua. De igual forma, en la ecuación se toman en cuenta otros factores que contribuyen a la resistencia, como las corrientes transversales o las secundarias (Knight y col., 2009). El CES fue desarrollado por diferentes organizaciones en el Reino Unido tales como la agencia ambiental, la agencia de ríos de Irlanda del Norte, el equipo de políticas de inundaciones, y el equipo de proyecto HR Wallingford/JBA. Knight y col. (2009) rea- 
lizaron varios estudios en diferentes países, especialmente en ríos ubicados en el Reino Unido, Argentina, Estados Unidos, etc; y en particular en dos áreas transversales de ríos montañosos en Ecuador y Nueva Zelanda (Knight y col., 2009).

El objetivo principal de este artículo es explorar la posibilidad de una mejor descripción transversal de la rugosidad en el escenario presentado por Knight y col. (2009) para el río Tomebamba en Ecuador. En este estudio se prueba la inclusión de escenarios de rugosidad adicionales y ecuaciones empíricas adicionales para predecir el parámetro de rugosidad. Los escenarios de rugosidad varían según el material del lecho del río. Algunas áreas contienen solo una zona de rugosidad y otras tres zonas de rugosidad: lado izquierdo, lado derecho y cauce. En ambos lados se utilizan los valores de rugosidad establecidos en el CES, que cuenta con una base de datos de valores de referencia de rugosidad ((Fisher y Dawson, 2003) como se cita en (Wallingford, 2013)). Sin embargo, para el cauce del río se utilizó un valor calibrado constante establecido por Knight y col. (2009) así como ecuaciones empíricas cuyos valores de rugosidad varían con la profundidad del agua debido a la presencia de las rocas, que afecta la resistencia debido a la interacción con el agua.

\section{Materiales y Métodos}

\subsection{Descripción del área}

Los datos del área transversal y la curva de descarga se tomaron de Knight y col. (2009). El área transversal a evaluar pertenece al río Tomebamba en Monay, Cuenca, Ecuador (Figura 1). El material del lecho está compuesto de rocas y grava, con un $\mathrm{D} 90=1,3$ metros. El alcance tiene una pendiente de 0,0176 y una anchura de $25 \mathrm{~m}$. La información sobre la toma de muestras del material del lecho está disponible en Bunte y Abt (2001) y Wolman (1954).

\subsection{Sistema de estimación de capacidad de trasporte (CES)}

El CES discretiza el ancho de un área transversal a intervalos constantes. La intersección del nivel de agua analizado con la sección transversal se considera como el primer y último elemento. En la Figura 2a, esos elementos son $y_{1}$ y $y_{1} 00$, y el número de elementos tomados por defecto es 100 . A continuación, el CES resuelve las ecuaciones promediadas en profundidad (RANS) a través del método de elemento finito (FEM), donde se obtiene el flujo unitario (q) para cada elemento $\left(y_{1}, y_{2}, \ldots, y_{100}\right)$. La selección de q se debe a sus propiedades de continuidad (Knight y col., 2009), y las condiciones límite en los elementos extremos son flujos unitarios iguales a cero. El flujo unitario se transforma entonces en velocidad de flujo promediada en profundidad (Ud en la Figura 2b) para cada elemento. Sin embargo, la distribución de velocidad cruzada no siempre está disponible para la validación del modelo, sino que más bien existe una curva de clasificación que relaciona el flujo con la profundidad del agua. Estos datos son obtenidos por el CES ya que hay una integración de velocidades promedias de profundidad para determinar un flujo promedio para toda el área. El CES repite todo el proceso anterior automáticamente para 25 profundidades, por lo que se obtiene una curva de descarga.

El parámetro de rugosidad en CES se realiza a través de un coeficiente denominado rugosidad unitaria (nl), que tiene las mismas unidades que el coeficiente de Manning. Este coeficiente evalúa los ríos del Reino Unido, donde hay una alta inmersión relativa y una profundidad de agua de 1 metro. Por lo tanto, este parámetro está relacionado únicamente con la disipación de la fricción. Dependiendo de la variación del material del lecho, la vegetación o las irregularidades en el área transversal, es posible determinar las zonas de rugosidad donde se asigna un determinado valor unitario de rugosidad. Por ejemplo, en la figura 3 las líneas rojas indican los límites de las zonas de rugosidad, en donde, por ejemplo, el banco izquierdo y derecho tienen un material de lecho de roca cubierto por vegetación, mientras que el lecho del río cuenta con rocas sin vegetación.

El software CES tiene un asesor de rugosidad que cuenta con valores típicos de resistencia unitaria para el material de la superficie, la vegetación y las irregularidades. Cada término se describe individualmente y se combina con una suma de raíces cuadradas. 


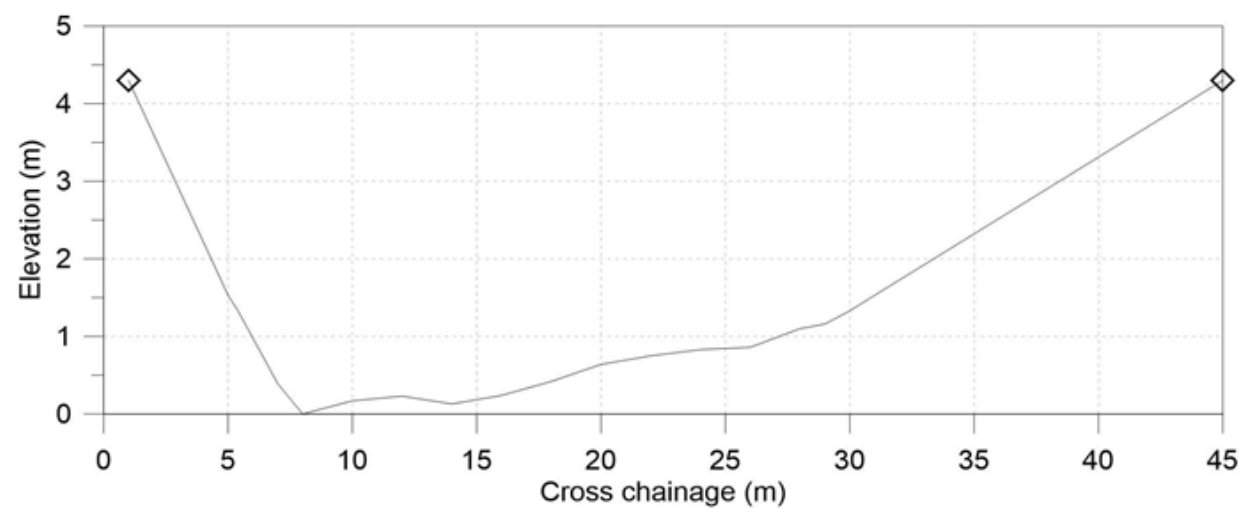

Figura 1. Área transversal del río Tomebamba. Datos adaptados de (Knight y col., 2009).

\section{3 Áreas}

En este trabajo se tomó como punto de partida el estudio del río Tomebamba presentado en Knight y col. (2009), el cual consistía en aplicar el CES a un río de montaña cuyo cauce está cubierto por rocas. Knight y col. (2009) analizaron tres casos en una sola área de rugosidad. En primer lugar, utilizaron los valores del asesor de rugosidad. En segundo lugar, utilizaron un valor unitario calibrado de rugosidad. Finalmente, consideraron un modelo de roca (ver Ecuación 1). Los datos de la validación consistieron en puntos de la curva. En nuestra investigación, ampliamos esos escenarios añadiendo dos zonas de rugosidad y modelos de roca adicionales para explorar la posibilidad de mejorar la predicción del modelo mejorando la descripción de rugosidad transversal. Los modelos de roca se utilizan para predecir los valores de rugosidad del cauce. Por otro lado, los valores de rugosidad del banco izquierdo y derecho fueron los presentados por el CES.

Los modelos de roca utilizados se basan en expresiones exponenciales y semilogarítmicas consideradas como enfoques tradicionales para la predicción de la resistencia (Zimmermann, 2010). El modelo de roca de Knight y col. (2009) es un modelo calibrado basado en datos de los ríos Tomebamba y Cuenca:

$$
f_{m c}=8 \cdot\left[5,75 \cdot \log \left(\frac{12 \cdot d_{m c}}{3 \cdot D_{90}}\right)\right]^{-2}
$$

Donde $d m c$ es la profundidad máxima del área transversal local [m], D90 es el percentil 90 de la distribución del tamaño del material [m]. Los detalles de este modelo se pueden encontrar en Abril y Knight (2004).

El modelo de roca de Romero, Revollo y Molina (2010) (Ecuación 2), obtenido a través de la regresión de datos de cinco ríos de montaña en Bolivia, da como resultado la siguiente ecuación con $S_{O}$ el lecho de roca:

$$
f=1,21 \cdot \ln \left(S_{O}\right)+6,259
$$

El modelo de roca de Bathurst (2002) se obtuvo con el análisis de veintisiete conjuntos de datos. En ese artículo, el autor se centra en los efectos de las variaciones entre áreas y en la resistencia. La Ecuación 3 se utiliza para las pendientes del cauce superiores a $0,8 \%$, donde $d$ es el promedio de profundidad de agua [m].

$$
\left(\frac{8}{f}\right)^{1 / 2}=3,1 \cdot\left(\frac{d}{D_{84}}\right)^{0,93}
$$

La razón del uso de D84 es que éste representa una vista 3-D del material del lecho del río. Sin embargo, al existir piletas o cascadas, esta altura de rugosidad parece no ser la apropiada (Lee y Ferguson, 2002; Maxwell y Papanicolaou, 2001; Aberle y Smart, 2003). Sin embargo, existen otros estudios en donde se utilizó el D84 con éxito para estas morfologías (Comiti y col., 2007). Si en un área se utiliza uno de los modelos de roca, el valor de $f$ se calculará y transformará en una rugosidad unitaria usando la Ecuación 4. Donde $g$ es la aceleración gravitacional $\left[\mathrm{m} \mathrm{s}^{-1}\right], n l$ rugosidad unitaria $\left[\mathrm{s} \mathrm{m}^{-1 / 3}\right], \mathrm{y} d$ es la 
a)

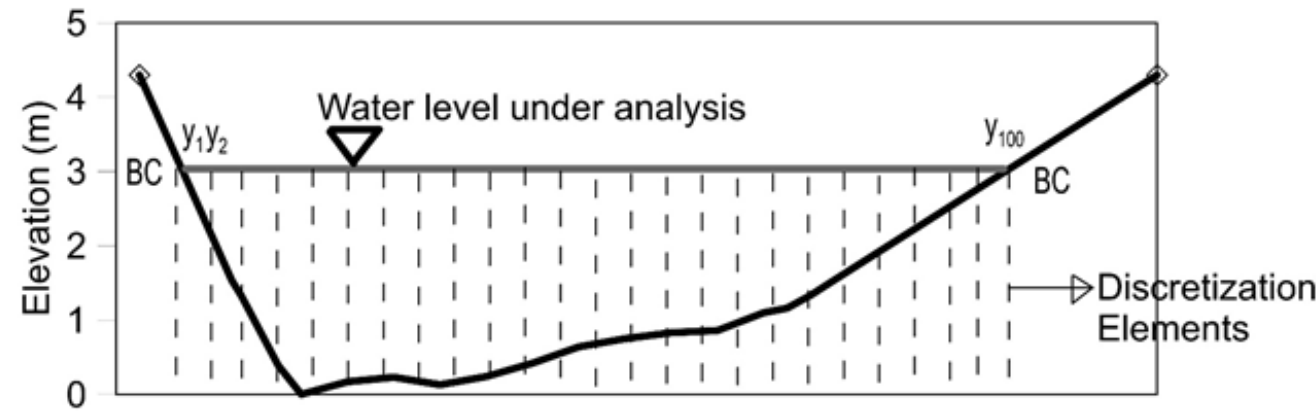

b)

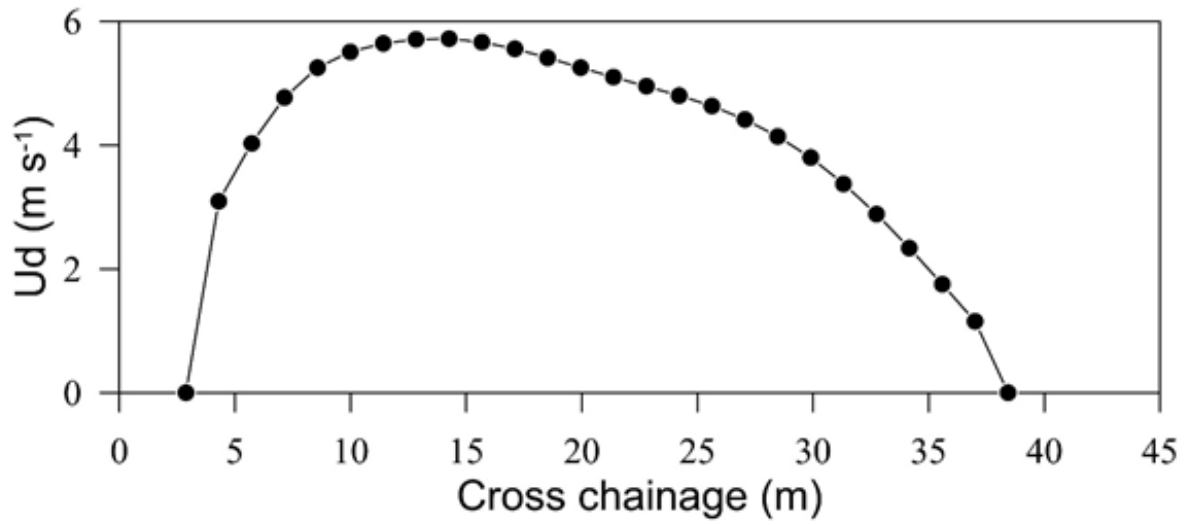

Figura 2. a) Discretización transversal. Datos de sección transversal adaptados de (Wallingford, 2013). b) Ejemplo de distribución de velocidad transversal correspondiente a la sección transversal ilustrada en a).

profundidad del agua [m]. Esta ecuación es el componente rugoso-turbulento de Colebrook-White.

$$
f=\frac{8 \cdot g \cdot n l^{2}}{d^{\frac{1}{3}}}
$$

El paquete CES calcula una curva de clasifica- ción (nivel de flujo-agua) para cada escenario descrito en la Tabla 1. Los resultados se comparan con los puntos medidos en las curvas de descarga a través de diferentes parámetros. Cada escenario se describe en la Tabla 1.

Tabla 1. Escenarios para el análisis de las curvas de descarga utilizando el paquete CES.

\begin{tabular}{cccc}
\hline Escenario & Descripción & Canal & Bancos \\
\hline 0 & Un escenario de rugosidad & Rugosidad unitaria calibrada de Knight y col. (2009) & Altura variable de la hierba + Grava fina \\
1 & Dos escenarios de rugosidad & Rugosidad unitaria calibrada de Knight y col. (2009) & Altura variable de la hierba + empedrado \\
2 & Dos escenarios de rugosidad & Rugosidad unitaria calibrada de Knight y col. (2009) & Modelo de roca de Knight y col. (2009) \\
3 & Un escenario de rugosidad & Modelo de roca de Knight y col. (2009) & Altura variable de la hierba + Grava fina \\
4 & Dos escenarios de rugosidad & Altura variable de la hierba + empedrado \\
5 & Dos escenarios de rugosidad & Modelo de roca de Knight y col. (2009) & Altura variable de la hierba + empedrado \\
6 & Dos escenarios de rugosidad & Modelo de roca de Bathurst (2002) & Altura variable de la hierba + empedrado \\
7 & Dos escenarios de rugosidad & Modelo de roca de Romero, Revollo y Molina (2010) & \\
\hline
\end{tabular}




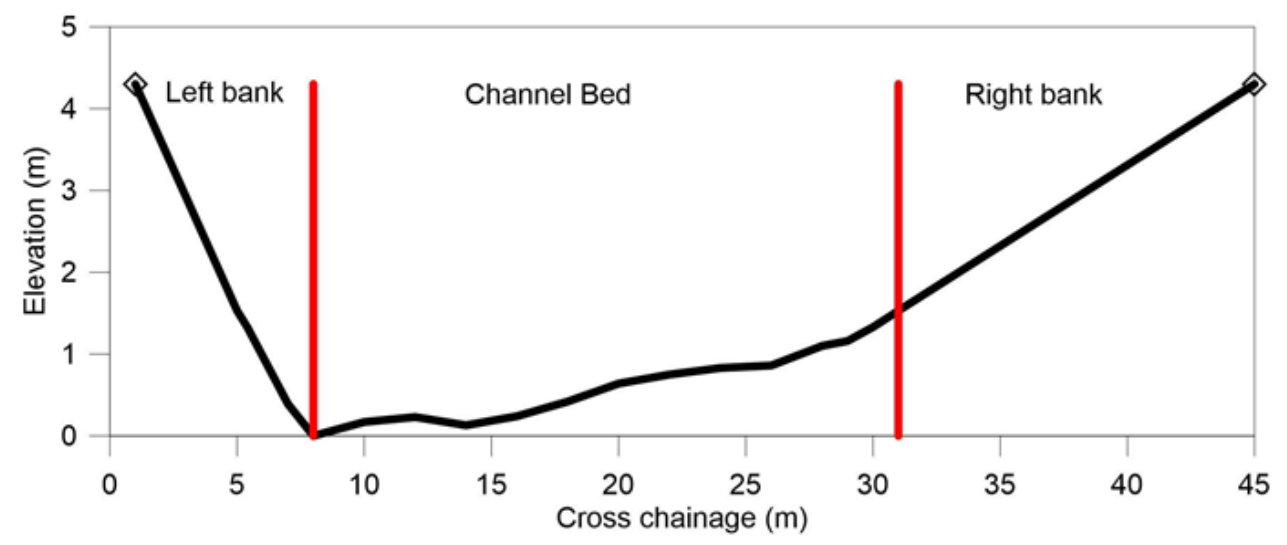

Figura 3. División de zonas de rugosidad.

\subsection{Parámetros de Ajuste Estadístico}

La cuantificación del rendimiento de cada escenario se realizó a través de índices estadísticos que abarcan en un solo número la calidad de predicción de un modelo en comparación con los datos de la validación. Sin embargo, cada parámetro muestra una proyección específica de la precisión del modelo en relación con los datos medidos (Chai y Draxler, 2014). A continuación, se puede encontrar una explicación de los parámetros utilizados.

MSE (raíz del error cuadrático medio) es un parámetro ampliamente utilizado en estudios meteorológicos y ambientales como por ejemplo de la calidad del aire o investigación climática (Willmott y Matsuura, 2005; Nayak y col., 2013; Ritter y Muñoz-Carpena, 2013; Chai y Draxler, 2014). Es una metodología cualitativa donde los errores más grandes del modelo tienen más peso que los más pequeños (Willmott y Matsuura, 2005; Chai y Draxler, 2014). De igual forma, Ferguson (2007) afirma que este parámetro es útil para estimar el rendimiento del modelo de valores altos, y tiene las mismas unidades que la variable bajo estudio.

Este parámetro es sensible a los valores atípicos, por lo que es importante eliminar los valores altos observados en la muestra (Chai y Draxler, 2014). La ecuación de RMSE se observa en la ecuación 5. Donde $S$ significa valores simulados, $O$ significa datos observados, y $N$ es el número de datos. Un modelo de ajuste perfecto tendrá un valor de RMSE de 0 .

$$
R M S E=\left(\frac{\sum_{i=1}^{n} S_{i}-O_{i}}{N}\right)^{0,5}
$$

EF (coeficiente de eficiencia) es un parámetro estadístico que mide la capacidad de estimación de la línea 1:1 entre los datos observados y medidos (Nash y Sutcliffe, 1970). Este parámetro se utiliza ampliamente para calcular la bondad de ajuste de los modelos debido a su flexibilidad y fiabilidad (McCuen y Cutter, 2006; Merz y Doppmann, 2006; Nayak y col., 2013). Sin embargo, es sensible al sesgo y los valores atípicos (McCuen y Cutter, 2006; Ritter y Muñoz-Carpena, 2013) y otorga mucha importancia a las diferencias entre los valores observados y los previstos como lo hace RMSE.

Cuando se analiza la ecuación 6, el numerador representa la variación inexplicable de los datos por el modelo, mientras que el denominador es la diferencia de los datos observados con respecto a la media (McCuen y Cutter, 2006). Ritter y MuñozCarpena (2013) otorgan en la Tabla 2 una gama de valores para EF y sus implicaciones para el rendimiento del modelo. Un EF de 1 significa que el modelo y los datos medidos encajan perfectamente; donde $\bar{O}_{i}$ es la media de las observaciones.

$$
E F=1-\left(\frac{\sum_{i=1}^{n}\left(O_{i}-S_{i}\right)^{2}}{\sum_{i=1}^{n}\left(O_{i}-\bar{O}_{i}\right)^{2}}\right)
$$


Tabla 2. Rendimiento del modelo basado en los valores de EF.

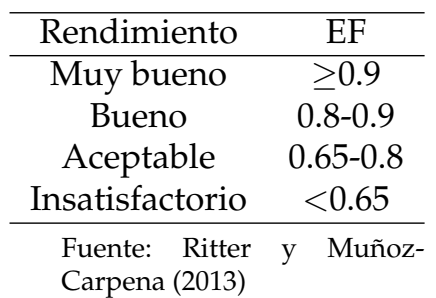

MAE (error absoluto medio) es el promedio de los errores (Ecuación 7). El RMSE es mayor que el $\mathrm{MAE}$, por lo que la magnitud de la diferencia indica la bondad del modelo (Alvarado, 2001). Este parámetro se utiliza ampliamente para las evaluaciones de los modelos (Chai y Draxler, 2014). (Chai y Draxler, 2014) consideran que este parámetro es un mejor indicador del error promedio que RMSE. Un modelo con un ajuste perfecto tiene un MAE de 0 .

$$
M A E=\frac{\sum_{i=1}^{n}\left|S_{i}-O_{i}\right|}{N}
$$

Error relativo (RE) (Ecuación 8), este parámetro representa el error relativo de la predicción tomando como base los datos medidos.

$$
R E=\frac{S_{i}-O_{i}}{O_{i}} \cdot 100 \%
$$

\section{Resultados}

\subsection{Curva de Clasificación}

La Figura 4a muestra las curvas de descarga cuando se utiliza una rugosidad unitaria calibrada. Esta figura muestra que la inclusión de zonas de rugosidad adicionales para los cauces conduce a una mejor aproximación de los datos medidos para flujos superiores a $20 \mathrm{~m}^{3} \mathrm{~s}^{-1}$.
La Figura 4b ilustra la sensibilidad del modelo hacia la selección del modelo de rugosidad. Por ejemplo, en los modelos 6 y 7, los nuevos modelos de rugosidad junto con áreas adicionales de rugosidad no mejoran la predicción de la curva de clasificación, sino que conducen a valores más bajos de altura del agua. Según la Figura $4 b$, el mejor escenario es el número 3, que utiliza una zona de rugosidad con el modelo de rugosidad de Knight y col. (2009).

\section{2 Índices Estadísticos}

La Tabla 3 presenta los resultados de los índices estadísticos, y las celdas en negrillas muestran el modelo con la coincidencia más cercana al valor óptimo para cada parámetro. El RMSE muestra que el escenario 3 (una zona de rugosidad, modelo de rugosidad de Knight y col. (2009)) es el mejor modelo. Además, el escenario con el menor error medio absoluto (MAE) fue el escenario 2 (dos zonas de rugosidad, descripción de cuenca: empedrado + hierba de altura variable). La magnitud de la diferencia entre estos dos índices muestra que el escenario 3 es el mejor para predecir la curva de clasificación en el río Tomebamba. Los valores EF confirman que los escenarios 2 y 3 son los mejores, con un buen rendimiento (ver Tabla 2). Además, la Tabla 4 presenta el error relativo con respecto a los datos medidos para cada flujo y para cada escenario. Esta tabla muestra que, para los valores de flujo bajo, el escenario 0 tiene menos diferencia con respecto a los datos medidos. Además, en estas magnitudes de flujo, la Tabla 4 representa el error relativo más alto para todos los escenarios. Sin embargo, para valores de flujo más altos, el escenario 2 tiene la diferencia relativa más baja en la mayoría de los datos. Este aspecto coincide con el mayor valor de MAE presentado en la Tabla 3 para el escenario 2.

Tabla 3. Valores de índices estadísticos para los escenarios evaluados.

\begin{tabular}{ccccccccc}
\hline & Escenario & Escenario & Escenario & Escenario & Escenario & Escenario & Escenario & Escenario \\
& 0 & 1 & 2 & 3 & 4 & 5 & 6 & 7 \\
\hline RMSE & 4,94 & 4,31 & 2,92 & $\mathbf{2 , 3 8}$ & 10,22 & 7,33 & 70,92 & 47,82 \\
MAE & 1,77 & 1,54 & $\mathbf{1 , 1}$ & 1,25 & 2,62 & 2,16 & 6,63 & 5,8 \\
RMSE-MAE & 3,16 & 2,77 & 1,81 & $\mathbf{1 , 1 3}$ & 7,6 & 5,17 & 64,3 & 42,03 \\
EF & 0,96 & 0,97 & $\mathbf{0 , 9 8}$ & $\mathbf{0 , 9 8}$ & 0,92 & 0,94 & 0,43 & 0,61 \\
\hline
\end{tabular}


a)

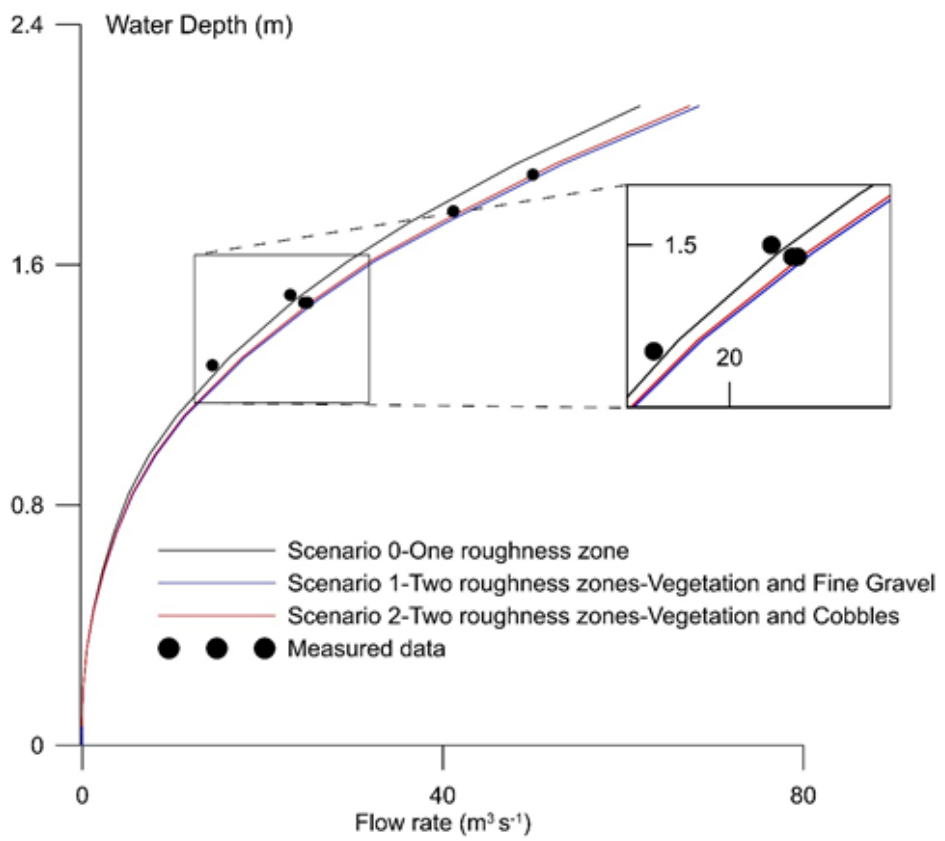

b)

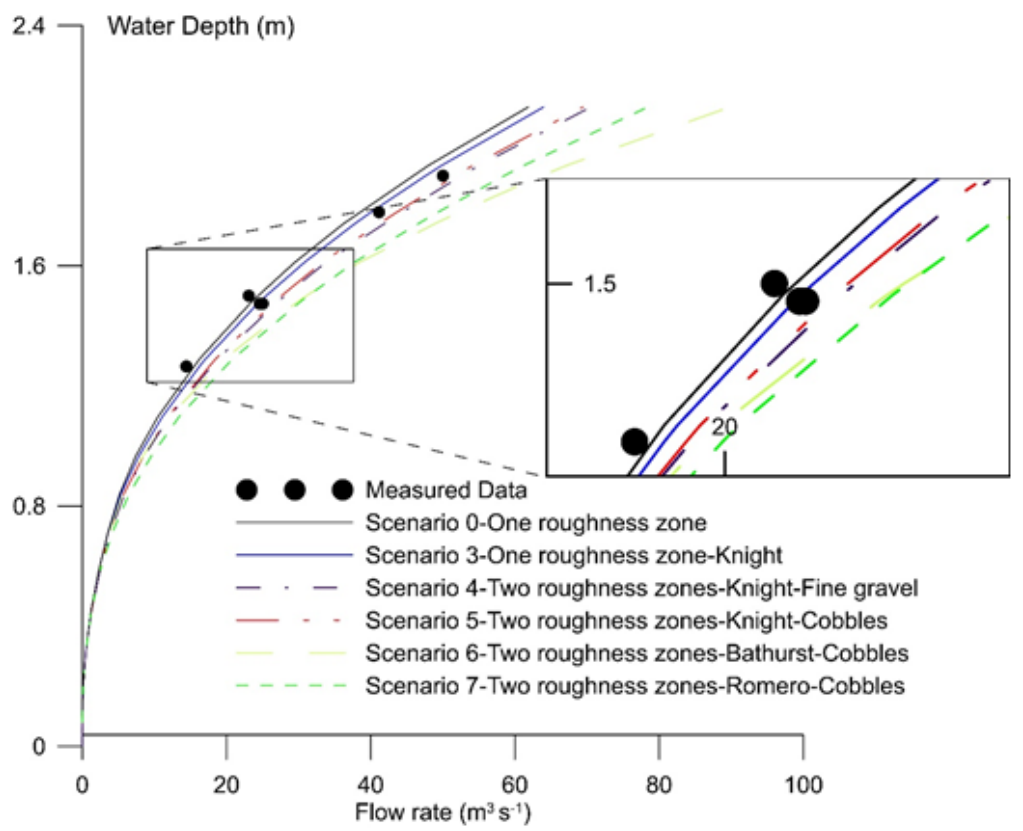

Figura 4. Curvas de descarga para diferentes escenarios. a) Mejor descripción del área del cauce. b) Modelo rugoso y mejor descripción del área del cauce.

\section{Discusión}

Dos escenarios han demostrado mejorar la capacidad de predicción de CES. De acuerdo con los parámetros el mejor escenario es el 3, que utiliza un modelo de roca con una sola zona de rugosidad. De igual forma, Knight y col. (2009) también reportaron que este método tuvo el mejor desempeño. El rendimiento del modelo puede deberse a una mejor descripción del patrón de resistencia en los ríos de montaña, donde los valores de resistencia cambian con la profundidad. Por otra parte, el modelo origi- 
nal tenía un valor fijo de resistencia para todas las profundidades del agua. El modelo clasificado como el segundo mejor es el que tiene tres escenarios de rugosidad: El lecho del canal tiene un parámetro de rugosidad calibrado y un refinamiento de la descripción de la cuenca. Este modelo es mejor en comparación con el escenario original, ya que muestra una descripción más realista de la resistencia a través del área transversal. Este modelo tiene un MAE mejor que el escenario 3. El MAE asigna el mismo peso a diferentes valores de error, mientras que el RMSE asigna un peso mayor a errores mayores. La diferencia entre ambos se puede observar en el escenario 3.

Tabla 4. Error relativo relacionado con la magnitud del flujo.

\begin{tabular}{ccccccccc}
\hline \multirow{Q}{*}{} & Escenario & Escenario & Escenario & Escenario & Escenario & Escenario & Escenario & Escenario \\
& 0 & 1 & 2 & 3 & 4 & 5 & 6 & 7 \\
\hline 14,42 & $\mathbf{7 , 1 2}$ & 18,63 & 16,62 & 12,67 & 24,18 & 22,10 & 29,35 & 37,68 \\
23,08 & $\mathbf{5 , 4 7}$ & 16,65 & 14,79 & 10,46 & 21,64 & 19,69 & 34,64 & 35,72 \\
24,62 & $-5,67$ & 4,57 & 2,86 & $\mathbf{- 1 , 2 8}$ & 9,04 & 7,21 & 19,84 & 21,55 \\
25,00 & $-7,12$ & 2,96 & $\mathbf{1 , 2 8}$ & $-2,80$ & 7,36 & 5,56 & 18,00 & 19,68 \\
41,15 & $-6,67$ & 3,83 & $\mathbf{2 , 1 3}$ & $-2,76$ & 7,72 & 5,94 & 26,87 & 19,80 \\
50,00 & $-8,38$ & 1,62 & $\mathbf{- 0 , 0 2}$ & $-4,74$ & 5,18 & 3,52 & 27,46 & 17,06 \\
\hline
\end{tabular}

La Tabla 4 muestra que la inclusión de un modelo de roca y/o zonas de rugosidad disminuye el error relativo a medida que aumenta el flujo. El agua y las rocas interactúan a menor flujo, generando flujos de inyección y ondas en las partículas que aumentan la resistencia al flujo, aumentando el parámetro de rugosidad (Jarrett, 1984; Bathurst, 2002). A medida que aumenta el flujo, este efecto se reduce, y el modelo parece ser más preciso para predecir los puntos en la curva de clasificación.

La rugosidad reportada por Bathurst (2002) y Romero, Revollo y Molina (2010) tienen los peores resultados de acuerdo con los parámetros utilizados. Al inicio, los datos con los que se derivaron ambos modelos parecían ser buenos para representar la rugosidad de las rocas en este río. Bathurst (2002) utilizó una cantidad importante de datos de la literatura, y Romero, Revollo y Molina (2010) utilizaron datos de ríos de la misma región andina que el río Tomebamba. El modelo de Knight y col. (2009) fue calibrado con datos de este río y otro de la misma ciudad, pero el aspecto crucial que influye en su previsibilidad podría deberse a su relación logarítmica para reflejar la rugosidad en comparación con la relación exponencial obtenida por Bathurst (2002). El modelo de Romero, Revollo y Molina (2010) puede fallar en la excesiva simplicidad de la relación, ya que no cuenta con un término que represente la variación de resistencia en el área, co- mo por ejemplo la inmersión relativa. Por lo tanto, el uso de una descripción inadecuada de rugosidad puede implicar errores importantes en las predicciones. Además, los resultados muestran que un factor de rugosidad calibrado para el lecho del canal y el uso del asesor de rugosidad para las cuencas pueden proporcionar buenos resultados de modelado cuando no se dispone de una rugosidad de roca apropiada.

\section{Conclusiones}

Esta investigación representa una extensión del estudio de caso del río Tomebamba, presentado por Knight y col. (2009). Se consideraron nuevos escenarios con diferentes modelos de roca y/o zonas de rugosidad. La previsibilidad se cuantifica a través de diferentes parámetros para verificar diferentes aspectos de los errores. Los datos utilizados para la validación se tomaron de una curva de descarga que se comparó con el rendimiento del modelo.

La mayoría de los parámetros muestran que la descripción de la rugosidad de Knight y col. (2009) proporciona los mejores resultados para igualar la curva de descarga medida en esta sección del río Tomebamba. Además, el escenario donde se agregó un valor de rugosidad calibrado y dos escenarios de rugosidad adicionales proporciona el mejor 
valor de MAE y el error relativo más bajo. Por lo tanto, el CES es capaz de predecir la curva de descarga en un río de montaña, pero se limita a predecir buenos resultados cuando la descripción de rugosidad toma en cuenta las condiciones locales, como lo hizo Knight y col. (2009). Por otra parte, el uso de una descripción de rugosidad obtenida de un conjunto de datos que no incluía el río Tomebamba ((Bathurst, 2002; Romero, Revollo y Molina, 2010) en este estudio) subestimó los niveles de agua que tienen un efecto potencialmente negativo durante la predicción de inundaciones. Por lo tanto, resulta necesario conocer los valores de rugosidad de cada área para obtener resultados de modelización apropiados, y cualquier ecuación empírica requiere una prueba previa antes de ser usada en un modelo.

\section{Financiación}

Esta investigación se desarrolló en el marco del proyecto "Desempeño y fiabilidad de modelos hidráulicos unidimensionales para la modelación de inundaciones en ríos de montaña" financiado por la Dirección de Investigación de la Universidad de Cuenca (DIUC) en el marco de la XI convocatoria de propuestas de investigación.

\section{Referencias}

Aberle, J. y G. Smart (2003). «The influence of roughness structure on flow resistance on steep slopes». En: Journal of hydraulic research 41.3, 259-269. Online:https://bit.ly/39LmnEv.

Abril, J. y D. Knight (2004). «Stage-discharge prediction for rivers in flood applying a depthaveraged model». En: Journal of Hydraulic Research 42.6, 616-629. Online:https : / / bit. ly / 3rqHkL7.

Alvarado, A. (2001). «Modelización hidrodinámica de canales de riego medianos». Graduate thesis. Cuenca, Ecuador: Universidad de Cuenca.

Bathurst, J. (1985). «Flow resistance estimation in mountain rivers». En: Journal of Hydraulic Engineering 111.4, 625-643. Online:https: / / bit.ly / 39LyxNB.

- (2002). «At-a-site variation and minimum flow resistance for mountain rivers». En: Journal of Hydrology 269.1-2, 11-26. Online:https: / / bit.ly / 3rlqop2.
Bhola, P., J. Leandro y M. Disse (2019). «Reducing uncertainties in flood inundation outputs of a two-dimensional hydrodynamic model by constraining roughness». En: Natural Hazards and Earth System Sciences 19.7, 1445-1457. Online:https://bit.ly/3oOlQWF.

Bunte, K. y S. Abt (2001). Sampling surface and subsurface particle-size distributions in wadable graveland cobble-bed streams for analyses in sediment transport, hydraulics, and streambed monitoring. US Department of Agriculture, Forest Service, Rocky Mountain Research Station.

Camp, J., D. Whyte y A. Shaw (2016). Vulnerabilities Due to Flooding Events. Inf. téc. National Center for Freight \& Infrastructure Research \& Education. Online:https:/ / bit.ly/3to8SCz.

Chai, T. y R. Draxler (2014). «Root mean square error (RMSE) or mean absolute error (MAE)? Arguments against avoiding RMSE in the literature». En: Geoscientific model development 7.3, 1247-1250. Online:https:/ / bit.ly/3aAXJpc.

Comiti, F. y col. (2007). «Field-derived relationships for flow velocity and resistance in high-gradient streams». En: Journal of hydrology 340.1-2, 48-62. Online:https: / / bit.ly/3jkapVt.

Douben, K. (2006). «Characteristics of river floods and flooding: a global overview, 1985-2003». En: Irrigation and Drainage: The journal of the International Commission on Irrigation and Drainage 55.1, S9-S21. Online:https://bit.ly/36FvqVC.

Ferguson, R. (2007). «Flow resistance equations for gravel-and boulder-bed streams». En: Water resources research 43.5. Online:https: / / bit . ly / 3tjdvxA.

Fisher, K. y H. Dawson (2003). Reducing Uncertainty in River Flood Conveyance - Roughness Review. Ed. por A. Peter. R \& D Coordinator, Flood Management Division.

Jarrett, R. D (1985). «Determination of roughness coefficients for streams in Colorado». Online:https://on.doi.gov/2Lg5BDZ.

Jarrett, R. (1984). "Hydraulics of high-gradient streams». En: Journal of hydraulic engineering 110.11, 1519-1539. Online:https : / / bit . ly / 3avk6fY.

Knight, D. y col. (2009). Practical channel hydraulics: Roughness, conveyance and afflux. First Ed. CRC Press.

Lee, A. y R. Ferguson (2002). «Velocity and flow resistance in step-pool streams». En: Geomorp- 
hology 46.1-2, 59-71. Online:https : / / bit . ly / 39NO4wB.

Marcus, W. y col. (1992). «An evaluation of methods for estimating Manning's $\mathrm{n}$ in small mountain streams». En: Mountain Research and Development 12.3, 227-239. Online:https:/ / bit.ly/36DhzPK.

Maxwell, A. y A. Papanicolaou (2001). «Steppool morphology in high-gradient streams». En: International Journal of Sediment Research 16.3, 380-390. Online:https:/ / bit.ly/2LfsZBw.

McCuen R.and Knight, Z. y A. Cutter (2006). «Evaluation of the Nash-Sutcliffe efficiency index». En: Journal of hydrologic engineering 11.6, 597-602. Online:.

Merz, J. y G. Doppmann (2006). Measuring Mountain Stream Discharge Using the Salt Dilution Method A practical guide. Kathmandu, Nepal: UoB PARDYP/ICIMOD e Hydrology Group.

Morvan, H. y col. (2008). «The concept of roughness in fluvial hydraulics and its formulation in 1D, 2D and 3D numerical simulation models». En: Journal of Hydraulic Research 46.2, 191-208. Online:https:/ / bit.ly/3tnlzxj.

Nash, J. y J. Sutcliffe (1970). «River flow forecasting through conceptual models part I-A discussion of principles». En: Journal of hydrology 10.3, 282-290. Online:https: / / bit.ly/3cRyeD9.

Nayak, P.C.a y col. (2013). «Rainfall-runoff modeling using conceptual, data driven, and wavelet based computing approach». En: Journal of Hydrology 493, 57-67. Online:https : / / bit.ly / 3tVZ0QQ.

Pagliara, S., R. Das e I. Carnacina (2008). «Flow resistance in large-scale roughness condition». En: Canadian Journal of Civil Engineering 35.11, 1285-1293. Online:https://bit.ly/3oI4dYF.
Papanicolaou, A., A. Bdour y E. Wicklein (2004). «One-dimensional hydrodynamic/sediment transport model applicable to steep mountain streams». En: Journal of Hydraulic Research 42.4, 357-375. Online:https:/ / bit.ly/36GrRhY.

Ritter, A. y R. Muñoz-Carpena (2013). «Performance evaluation of hydrological models: Statistical significance for reducing subjectivity in goodness-of-fit assessments». En: Journal of Hydrology 480, 33-45. Online:https : / / bit . ly / 3cG2SPI.

Romero, M., N. Revollo y J. Molina (2010). «Flow resistance in steep mountain rivers in Bolivia». En: Journal of Hydrodynamics 22.5, 702-707. Online:https://bit.ly/36FiizS.

Wallingford, H. (2013). «Reducing uncertainty in river flood conveyance, Phase 2 Conveyance Manual». Online:https://bit.ly/3pQBIJx.

Willmott, C. y K. Matsuura (2005). «Advantages of the mean absolute error (MAE) over the root mean square error (RMSE) in assessing average model performance». En: Climate research 30.1, 79-82. Online:https://bit.ly/3rjjq3Z.

Wohl, E. (2000). Mountain Rivers. Washington, D. C: American Geophysical Union (Water Resources Monograph).

Wolman, M. (1954). "A method of sampling coarse river-bed material». En: EOS, Transactions American Geophysical Union 35.6, 951-956. Online:https: //bit.ly/36Do05m.

Zimmermann, André (2010). «Flow resistance in steep streams: An experimental study». En: $W a-$ ter Resources Research 46.9. Online:https: / / bit . ly/3jhFrxl. 